冬 J.B.METZLER 
Andrea Frank, Stefanie Haacke und Swantje Lahm

\section{Schlüsselkompetenzen: Schreiben in Studium und Beruf}

Mit Abbildungen und Graphiken

Verlag J.B. Metzler Stuttgart · Weimar 


\section{Die Autorinnen}

Andrea Frank (geb. 1959), Studium der Pädagogik und Soziologie an der Uni Bielefeld, Promotion, 1993 Gründung des ersten Schreiblabors an einer dt. Universität, 1993-2005 Referentin des Prorektors für Studium und Lehre, seit 2005 Leiterin des neuen Arbeitsbereichs Beratung für Studium, Lehre \& Karriere an der Universität Bielefeld.

Stefanie Haacke (geb. 1961), Studium der Philosophie, Religionswissenschaft und Kunstgeschichte an der FU Berlin, seit 1998 Mitarbeiterin im Schreiblabor. Arbeitsschwerpunkte: Unterstützung von Lehrenden bei der Konzeption von schreibintensiven Lehrveranstaltungen, Ausbildung von Schreibtrainer/innen, Schreibberatung.

Swantje Lahm (geb. 1974), Studium der Geschichte, Soziologie und Osteuropäischen Studien an der Uni Bielefeld, seit 2003 Mitarbeiterin im Schreiblabor. Arbeitsschwerpunkte: Unterstützung von Lehrenden bei der Konzeption von schreibintensiven Lehrveranstaltungen, Ausbildung von Schreibtrainer/innen, Schreibberatung.

Bibliografische Information Der Deutschen Nationalbibliothek Die Deutsche Nationalbibliothek verzeichnet diese Publikation in der Deutschen Nationalbibliografie; detaillierte bibliografische Daten sind im Internet über $<$ http://dnb.d-nb.de > abrufbar.

ISBN 978-3-476-02166-3

ISBN 978-3-476-05053-3 (eBook)

DOI 10.1007/978-3-476-05053-3

Dieses Werk einschließlich aller seiner Teile ist urheberrechtlich geschützt. Jede Verwertung außerhalb der engen Grenzen des Urheberrechtsgesetzes ist ohne $\mathrm{Zu}$ stimmung des Verlages unzulässig und strafbar. Das gilt insbesondere für Vervielfältigungen, Übersetzungen, Mikroverfilmungen und die Einspeicherung und Verarbeitung in elektronischen Systemen.

() 2007 Springer-Verlag GmbH Deutschland Ursprünglich erschienen bei J.B. Metzler'sche Verlagsbuchhandlung und Carl Ernst Poeschel Verlag GmbH in Stuttgart 2007

www.metzlerverlag.de info@metzlerverlag.de 


\section{Vorwort}

Ist schon alles gesagt, nur noch nicht von allen?

nach

Karl Valentin

Schon wieder ein Buch zum wissenschaftlichen Schreiben? Sind nicht schon genügend Ratgeber auf dem Markt? Haben wir es einmal mehr mit dem "Valentin-Phänomen« zu tun, wonach schon alles gesagt ist - nur noch nicht von allen?

Was uns dazu bewogen und motiviert hat, dieses Buch zu schreiben, sind zum einen unsere langjährigen praktischen Erfahrungen in der Beratung von Studierenden und Lehrenden zur Förderung der Schreibkompetenz im Studium. Sie führen uns immer wieder vor Augen, welche Chancen das Schreiben im Studium gerade für Geistes- und Sozialwissenschaftler/innen birgt und wie wenig sie genutzt werden. $\mathrm{Zu}$ viele Studierende verlassen die Universität und behalten das Schreiben im Studium als lästige und unbefriedigende Tätigkeit ohne jeden Sinn in Erinnerung, manchmal sogar als so qualvoll, dass sie nie wieder gerne schreiben. Das ist schade. Und so sollte es nicht sein.

Zum anderen sind es unsere Beobachtungen dazu, wie sich die institutionellen Rahmenbedingungen für das Schreiben im Studium in den letzten Jahren - insbesondere mit der Einführung der neuen Bachelorund Masterstudiengänge - verändert haben: Als das Schreiblabor 1993 an der Universität Bielefeld gegründet wurde, geschah dies vor dem Hintergrund einer Studien- und Lehrsituation, in der es - zumindest in den Geistes- und Sozialwissenschaften - wenige inhaltliche Vorgaben und so gut wie keine organisatorisch-strukturellen Reglementierungen gab. Zwar existierten Studienordnungen, aber diese gaben kaum eine verbindliche Orientierung für das individuelle Studieren. Ein geisteswissenschaftliches Magisterstudium bot deshalb viel Zeit, sich intensiv mit Themen $\mathrm{zu}$ beschäftigen, sich in Lektüre $\mathrm{zu}$ vertiefen und dabei eigene Schwerpunkte zu setzen. Die Kehrseite dieser akademischen Freiheit im Studium bestand darin, dass ihr längst nicht alle gewachsen waren. Viel zu viele Studierende brachen ihr Studium ohne Abschluss $\mathrm{ab}$, andere benötigten viel mehr Zeit als vorgesehen, um es erfolgreich zu beenden. Die fehlende Zeitbegrenzung förderte geradezu das Ausufern von Themen von Haus- und Abschlussarbeiten. Die meisten der Studierenden, die in dieser Zeit das Schreiblabor aufsuchten, waren in höheren Semestern und bereits seit vielen Monaten mit ihren schriftlichen Arbeiten beschäftigt.

Mit der Einführung der Bachelorstudiengänge 2002 veränderten sich die Rahmenbedingungen für das Studium und damit auch die Fragen der Studierenden: Viele, die das Schreiblabor heute aufsuchen, sind in den Anfangssemestern, stehen aufgrund studienbegleitender Prüfungen unter Druck, sind mit einer Vielzahl unterschiedlicher Aufgaben gleichzeitig konfrontiert, hatten noch gar keine Gelegenheit, sich 
inhaltlich zu orientieren - und scheinen vor allem eines nicht zu haben: Zeit. Das Problem heißt nicht mehr: Wie geht man mit zu viel Freiheit um?, sondern eher: Wie kann man sich einen strukturierten Rahmen schaffen, um ein Schreibprojekt in einer angemessenen Zeit zu Ende zu bringen? Wie findet man Freiräume, in denen man sich auf sein Thema einlassen und kreativ werden kann? Wir möchten die Studierenden, die unser Buch lesen, ermuntern, sich solche Freiräume zu schaffen und das Schreiben im Studium für ihre Entwicklung zu nutzen.

Das Schreiben im Studium führt direkt in die Wissenschaft, denn wer Studienarbeiten schreibt, lernt mit fachlichen Inhalten, Methoden, Fragen und Problemen umzugehen. Schreiben führt aber auch in die Praxis. Es schult für jede Art von Handeln, das nicht im bloßen Ausführen von Anweisungen oder Reproduzieren von Bekanntem besteht.

Mit unserem Buch möchten wir Studierende dabei unterstützen, das $\mathrm{zu}$ suchen, $\mathrm{zu}$ entdecken und einzufordern, was ein Studium zu etwas anderem macht als eine Fortsetzung von Schule unter schlechteren Bedingungen.

Hinter einem fertigen Text stehen sehr viel mehr Menschen als die Autor/innen selbst. Entsprechend haben wir vielen für Unterstützung, Feedback und Rat zu danken. Vor allem danken wir den Studierenden, die unser Manuskript gelesen haben. Ihr Feedback war für unsere Arbeit sehr wichtig. Ein großer Dank geht auch an die zahlreichen berufstätigen Geisteswissenschaftler/innen, die uns mitgeteilt haben, ob und wie sie das Schreibenlernen im Studium für das Schreiben in ihren Berufen in und außerhalb der Wissenschaft nutzen konnten. Natürlich haben wir auch denjenigen zu danken, von denen wir gelernt und mit denen wir entwickelt haben, was wir in diesem Buch präsentieren, vor allem unseren Kolleg/innen. Und nicht zuletzt denen, die uns den Rücken freigehalten, uns getröstet und ertragen und uns geholfen haben, auch Konflikte durchzustehen. Wir bedanken uns also bei: Stella Büker, Hans-Volkmar Findeisen, Christine Freese, Angelika Fritsche, Ingrid Furchner, Paula Gillespie, Mareike Gronich, Ruth Großmaß, Silvia Herb, Keith Hjortshoj, Dörte Husmann, Harvey Kail, Karl Klingenberg, Hans-Martin Kruckis, Katrin Lehnen, Thomas Lüttenberg, Johannes Lüttenberg, Klaus Luther, Helen Menges, Sigurd Müller, Guido Nottbusch, Rolf Parr, Iliana Popova, Eva Reichmann, Michael Reiter, Gregor Rohmann, Gabriela Ruhmann, Ingrid Scharlau, Kirsten Schindler, Jana Schuster, Judith Seha, Ursel Sickendiek, Bianca Sievert, Patricia Skorge, Caroline Striewisch, Christina Tente, David Wegener, Thomas Welskopp, Holger Wiethäuper, Uwe Zimmer. 


\section{Inhaltsverzeichnis}

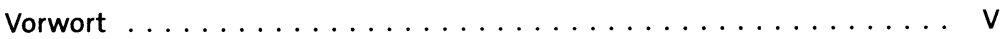

1. Einleitung .......................... 1

1.1 Schreiben im Studium . . . . . . . . . . . . . . 3

1.2 Vom Schreiben im Studium zum beruflichen Schreiben . . . . . . . . . 6

1.3 Schreiben ist eine individuelle Angelegenheit $\ldots \ldots \ldots \ldots \ldots$

1.4 Wie Sie dieses Buch nutzen können . . . . . . . . . . . . 11

2. Phasen im Schreibprozess . . . . . . . . . . . . . . . 13

2.1 Themenfindung $\ldots \ldots \ldots \ldots \ldots \ldots \ldots \ldots \ldots$

2.1.1 Sich vorgegebene Themen sinteressant arbeiten $4 \ldots \ldots \ldots \ldots$

2.1.2 'Eigene، Themen finden und das ,Eigener in Themen bewältigen . . . . 17

2.1.3 Themenfindung methodisch angehen . . . . . . . . . . 19

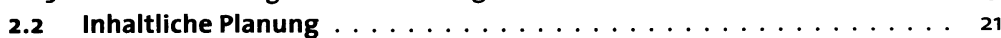

2.2.1 Themenformulierung und Themeneingrenzung . . . . . . . . 24

2.3 Suchen, Finden, Auswerten von Material und Literatur . . . . . . . 31

2.3.1 Recherchieren ....................... 32

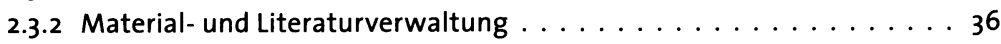

2.3.3 Material und Literatur auswerten: Exzerpte verfassen . . . . . . . . . 39

2.3.4 Literatur in Studienarbeiten: Zwei typische Missverständnisse . . . 42

2.4 Den roten Faden finden: Material und Gedanken strukturieren . . . . . 45

2.4.1 Gute Rahmenbedingungen für das Strukturieren schaffen . . . . . . . 47

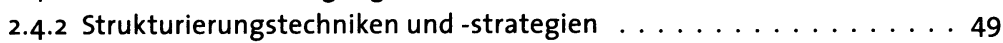

2.5 Die Rohfassung schreiben $\ldots \ldots \ldots \ldots \ldots \ldots \ldots \ldots \ldots \ldots$

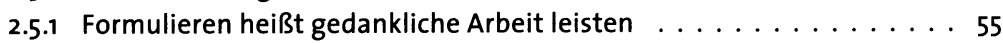

2.5.2 Über Gelesenes schreiben - die Literatur in Ihrem Text . . . . . . . . . . 60

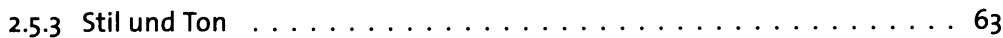

2.6 Textüberarbeitung: Schreiben heißt Umschreiben . . . . . . . 65

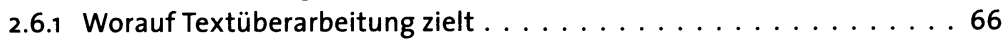

2.7 Endredaktion und Abgeben $\ldots \ldots \ldots \ldots \ldots \ldots \ldots \ldots \ldots$

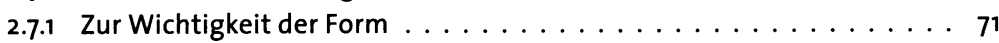

3. Schreibprojekte managen - Allein und mit anderen . . . 72

3.1 Arbeitsplanung und Zeitmanagement $\ldots \ldots \ldots \ldots \ldots \ldots \ldots \ldots$

3.1.1 Tipps zur Zeitplanung $\ldots \ldots \ldots \ldots \ldots \ldots \ldots \ldots$

3.2 Das Schreiben vor dem Schreiben . . . . . . . . . . . . . 78

3.2.1 Schreiben heißt Denken, Planen, Klären ... . . . . . . . . . . . . 79

3.3 Stockungen im Schreibprozess und was dahinterstecken kann . . . . . 88

3.3.1 Wenn Sie auf der Stelle treten . . . . . . . . . . . . . 90

3.3.2 Schreiben unter Druck . . . . . . . . . . . . . . . . 94

3.4 Feedback und Beratung $\ldots \ldots \ldots \ldots \ldots \ldots \ldots \ldots \ldots$

3.4.1 Organisation von Feedback: Allgemeine Hinweise $\ldots \ldots \ldots . .98$ 
3.4.2 Feedback geben: Was zu beachten ist . . . . . . . . . . . . . . . . 101

3.4.3 Feedback und Rat vom Betreuer/von der Betreuerin . . . . . . . . . . . 102

3.4.4 Feedback in Kolloquien, bei Tagungen und Konferenzen . . . . . . . . . . 103

3.5 Schreiben im Team ... . . . . . . . . . . . . . . . . 104

3.5 .1 Zusammenarbeit organisieren ...................... 106

\section{Flexibel umgehen mit Textarten}

und Darstellungsformen . . . . . . . . . . . . 112

4.1 Niemand fängt bei Null an . . . . . . . . . . . . . . . 113

4.2 Texte konzipieren und gestalten . . . . . . . . . . . . . 116

4.2.1 Kontext: Der Blick auf das Ganze . . . . . . . . . . . . . . . . . . . . 120

4.2.2 Inhalt und Thema: Was schreibe ich? . . . . . . . . . . . . . . 121

4.2.3 Ziel und Funktion: Wozu schreibe ich? . . . . . . . . . . . . . . . 122

4.2.4 Adressaten: Für wen schreibe ich? . . . . . . . . . . . . . . . . . . . . . . . . . . . . . . . . . .

4.2.5 Rolle und Haltung: Als wer schreibe ich? . . . . . . . . . . . . . . . . 125

4.2.6 Form: Textkonventionen berücksichtigen . . . . . . . . . . . . . . . 127

4.3 Anforderungen klären und Vorbilder suchen . . . . . . . . . . . . . 128

5. Textarten und Darstellungsformen . . . . . . . . . 132

5.1 Die wissenschaftliche Abhandlung:

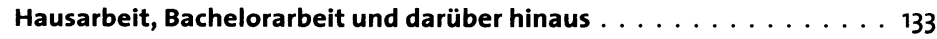

5.1.1 Hausarbeiten schreiben im Studium - wozu? . . . . . . . . . . . . 134

5.1.2 Grundsätze wissenschaftlicher Kommunikation in Hausarbeiten . . . 135

5.1.3 Die Teile der Hausarbeit: Standards und Spielräume . . . . . . . . . 138

5.1.4 Die Sprache in Hausarbeiten - Einige Empfehlungen . . . . . . . . . . . . . . . . . . . . . . . . . . . . . . .

5.1.5 Die Bachelorarbeit . . . . . . . . . . . . . . . . . . . . 141

5.1.6 Zeitmanagement: das A\&O bei der Bachelorarbeit . . . . . . . . 143

5.1.7 Masterarbeit und Doktorarbeit: die ,Nächstgrößeren، . . . . . . 144

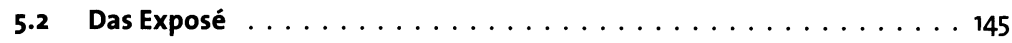

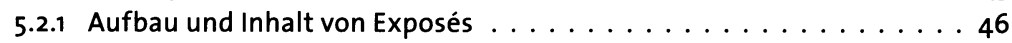

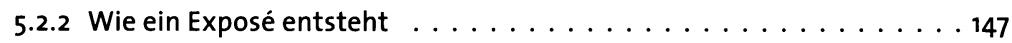

5.3 Die Mitschrift . . . . . . . . . . . . . . . . 151

5.3.1 Mitschriften können verschiedene Funktionen haben . . . . . . 152

5.3.2 Mitschriften können unterschiedliche Formen haben . . . . . . 152

5.3 .3 Wie eine Mitschrift entsteht . . . . . . . . . . . . . . . 154

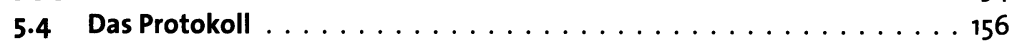

5.4.1 Das Verlaufsprotokoll . . . . . . . . . . . . . . . . . . . . . 157

5.4 .2 Das Ergebnisprotokoll . . . . . . . . . . . . . . . . . 160

5.4 .3 Das Seminarprotokoll . . . . . . . . . . . . . . . . . . . 161

5.5 Schreiben fürs Sprechen: Manuskripte für Referat und Vortrag . . . . 164

5.5 .1 Der Aufbau eines Vortrags . . . . . . . . . . . . . . . . 165

5.5 .2 Manuskripttypen . . . . . . . . . . . . . . . . . 167

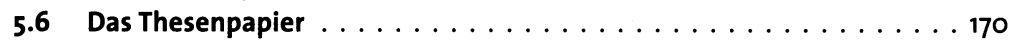

5.6 .1 Form und Sprache $\ldots \ldots \ldots \ldots \ldots \ldots \ldots \ldots \ldots \ldots$

5.6 .2 Wie eine These entsteht $\ldots \ldots \ldots \ldots \ldots \ldots \ldots$ 
5.7 Der Essay . . . . . . . . . . . . . . . . . . . . 174

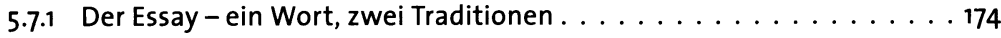

5.7.2 Der Essay als universitäre Übungsform . . . . . . . . . . . . . . . . . . . . . . . . . . . . . . . . . . . . . . .

5.7.3 Was zeichnet einen guten Essay aus? . . . . . . . . . . . . . . . . . . . . . . . . . . . . . . .

5.7 .4 Wie ein Essay entsteht . . . . . . . . . . . . . . . . . . . . . . . . . . . . . . . . . . . . . . . .

5.8 Die Klausur . . . . . . . . . . . . . . . . . . . . . . 180

5.8.1 Wie Sie sich auf eine Klausur vorbereiten können . . . . . . . . . . . . 180

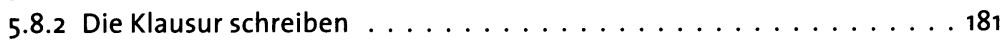

5.9 Die Rezension . . . . . . . . . . . . . . . . . . . . . . 183

5.9 .1 Informieren, kontextualisieren, kritisieren . . . . . . . . . . 184

5.9 .2 Wie eine Rezension entsteht . . . . . . . . . . . . . . . . . . . . . . . . . . . . . . . . . . . . . . . . . . . .

5.10 Der Praktikumsbericht . . . . . . . . . . . . . . . . . . 187

5.10 .1 Das Schreiben des Berichts vorbereiten . . . . . . . . . . . . . . 188

5.10 .2 Erfolgstagebuch führen . . . . . . . . . . . . . . . . . 189

5.10.3 Den Praktikumsbericht schreiben . . . . . . . . . . . . . . . . . . . . 191

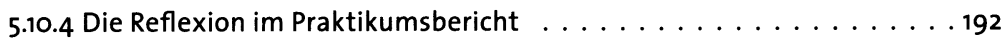

5.11 Schreiben für das Internet . . . . . . . . . . . . . . . . . 194

5.11 .1 Aufbau und Oberfläche . . . . . . . . . . . . . . . . . . . . . . . . . . . . . . . . . . . . . . . . . . . . . . . .

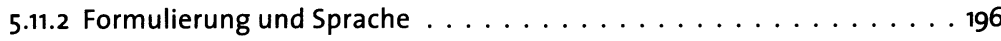

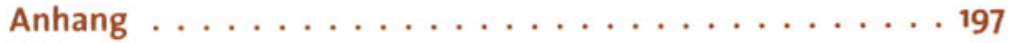

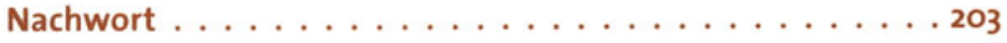

Literaturverzeichnis . . . . . . . . . . . . . 204 